\title{
Regular Problems for Semilinear Hyperbolic Type Equations
}

\author{
Dong-Gun Park, Jin-Mun Jeong and Han-Geul Kim
}

\begin{abstract}
In this paper we establish the wellposedness and regularity properties of solutions of Cauchy problems for semilinear hyperbolic equations of second order with unbounded principal operators. An example illustrating how our results apply is given.

Mathematics Subject Classification (2000). Primary 35F25; Secondary 35K55.

Keywords. Hyperbolic equations of second order, semilinear equation, regularity, analytic semigroup.
\end{abstract}

\section{Introduction}

This paper is concerned with regularity of solutions for an abstract semilinear wave equation:

$$
\left\{\begin{array}{l}
\frac{\partial^{2}}{\partial t^{2}} u(t, x)-\sum_{i, j=1}^{n} \frac{\partial}{\partial x_{j}}\left(a_{i j}(t, x) \frac{\partial u}{\partial x_{i}}\right)+c(t, x) u= \\
=\int_{0}^{t} \sum_{i=1}^{n} \frac{\partial}{\partial x_{i}} \sigma_{i}(s, \nabla u(s, x)) d s+h(t), 0 \leq t, \quad x \in \Omega, \\
u(t, x)=00 \leq t, \quad x \in \partial \Omega, \\
u(0, x)=u_{0}(x), \quad \frac{\partial}{\partial t} u(0, x)=u_{1}(x), \quad x \in \Omega .
\end{array}\right.
$$

The problem (1.1) is formulated as the following

$$
\left\{\begin{array}{l}
u^{\prime \prime}(t)+A(t) u(t)=G(t, u(t))+f(t) \\
u(0)=u_{0}, \quad u^{\prime}(0)=u_{1} .
\end{array}\right.
$$

This kind of equations arise naturally in population models, in materials science, in biology, engineering, etc. (see, for instance, $[2,18]$ and the bibliography therein). Starting from the pioneering results about the linear case, the regularity for solutions of Cauchy problems for linear hyperbolic equations of second order with boundary conditions has been studied by Ikawa [5]. As for second order nonlinear functional evolutions, Kalsatos and Markov in [8] have analyzed some questions on 
existence of solutions for functional differential inclusions of second order in time and in [3] proved them in the case where a damping term is added. Kato $[9,10]$ was the first to make a successful attack on the hyperbolic type problem. In recent papers, which generalize Kato's linear theory, Tanaka [16] has proved wellposedness of the first order nonautonomous abstract Cauchy problems for strongly measurable families, under a new type of quasi-stability condition from the viewpoint of the theory of finite difference approximations and Kobayashi [12] under strong continuity of $A$.

An example of parabolic type problems in which the nonlinear term is Lipschitz continuous but the mild solution of the equation is not a strong solution can be found in Webb [17]. We note that Lipschitz continuity of nonlinear term can be replaced by accretiveness and one still obtains, under suitable conditions, global solutions of the parabolic type equation, see Chapter 8 of Martin [14]. Recently, Kobayashi et al. [11] introduced the notion of semigroups of locally Lipschitz operators used to represent the mild solutions of the Cauchy problem for semilinear evolution equations. The regularity for the semilinear heat equations has been developed as in Section 4.3.1 of Barbu [1] and in [6].

In this paper, we propose an approach different from that of earlier works (see $[4,5,17])$ to study mild, strong, and classical solutions of Cauchy problems. We allow implicit arguments about $L^{2}$-regularity results for semilinear hyperbolic equations under more general hypotheses of nonlinear term $G$. These consequences are obtained by showing that results of the linear cases to those of [5] on the $L^{2}$-regularity remain valid under the above formulation of the semilinear problem (SE).

In Section 2, we give some basic results and introduce the main tools of our scheme. We adhere to the construction of an evolution system for the initial value problem (SE) with an unbounded operator $A(t)$ carried out by Kato in $[9,10]$. We assume that for each $t \geq 0, A(t)$ is the infinitesimal generator of an analytic semigroup together with some continuity conditions on the family of bounded operators $A(t) A(s)^{-1}$. Section 3 is devoted to the study of the regularity of solutions of the linear wave equations in Gelfand triple spaces. Subsequently, our construction of a local solution of the nonlinear equation (SE) is essentially based on [7]. We will show the energy inequalities for our problem (SE) with the aid of estimate of $L^{2}$ type of the solutions. These play an important role in the proof of the existence of global solutions and in that of the regularity of solutions. Finally, in Section 4, a possible extension of the given equation (1.1) is discussed.

\section{Preliminaries}

Let $H$ be a complex Hilbert space with inner product $(\cdot, \cdot)$ and norm $|\cdot|$. Let $V$ be embedded in $H$ as a dense subspace with inner product and norm by $((\cdot, \cdot))$ and $\|\cdot\|$, respectively. By considering $H=H^{*}$, we may write $V \subset H \subset V^{*}$ where $V^{*}$ denotes the dual space of $V$; its inner product and norm will be denoted by 
$(\cdot, \cdot)_{*}$ and $\|\cdot\|_{*}$, respectively. For $l \in V^{*}$ we denote $(l, v)$ by the value $l(v)$ of $l$ at $v \in V$. The norm of $l$ as element of $V^{*}$ is given by

$$
\|l\|_{*}=\sup _{v \in V} \frac{|(l, v)|}{\|v\|} .
$$

Therefore $V$ has a topology stronger than that of $H$ and we may assume that

$$
\|u\|_{*} \leq|u| \leq\|u\|, \quad \forall u \in V .
$$

Let $a(t ; u, v)$ be quadratic form defined on $V \times V$ and let us also make the following assumptions:

i) $a(t ; u, v)$ is bounded, uniformly Lipschitz continuous and $\frac{d}{d t} a(t ; u, v)$ is strongly continuous with respect to $t$, i.e., there are some positive constants $c_{0}, c_{1}$ such that

$$
\begin{aligned}
|a(t ; u, v)| & \leq c_{0}\|u\|\|v\| \\
|a(t ; u, v)-a(s ; u, v)| & \leq c_{1}|t-s|\|u\|\|v\|, \\
\left|\frac{d}{d t} a(t ; u, v)\right| & =|\dot{a}(t ; u, v)| \leq c_{1}\|u\|\|v\| ;
\end{aligned}
$$

ii) $a(t ; u, v)$ is symmetric, i.e.,

$$
a(t ; u, v)=\overline{a(t ; v, u)} ;
$$

iii) $a(t ; u, v)$ satisfies the Gårding's inequality, i.e.,

$$
\operatorname{Re} a(t ; u, u) \geq \delta\|u\|^{2}, \quad \delta>0 .
$$

Lemma 2.1. Let $A(t)$ be the operator associated to the quadratic form a $(t ; u, v)$, i.e.

$$
a(t ; u, v)=(A(t) u, v), \quad \text { for all } u, v \in V .
$$

Then $A(t)$ is an isomorphism from $V$ onto $V^{*}$ and for all $u \in V$, we have

$$
\delta\|u\| \leq\|A(t) u\|_{*} \leq c_{0}\|u\| .
$$

Proof. From assumptions i), iii) it follows that

$$
\|A(t) u\|_{*}=\sup _{v \in V} \frac{|(A(t) u, v)|}{\|v\|}=\sup _{v \in V} \frac{|a(t ; u, v)|}{\|v\|} \leq c_{0}\|u\|,
$$

and

$$
\|A(t) u\|_{*} \geq \frac{|(A(t) u, u)|}{\|u\|}=\frac{|a(t ; u, u)|}{\|u\|} \geq \delta\|u\|,
$$

which proves (2.1) and the lemma.

The restriction of $A(t)$ to

$$
D\left(A_{H}(t)\right)=\{u \in V \mid A(t) u \in H\}
$$

is denoted by $A_{H}(t)$. 
It is well known that $D\left(A_{H}(t)\right)$, which is structured as a Hilbert space with the norm $\|u\|_{D\left(A_{H}(t)\right)}=\left|A_{H}(t) u\right|$, is dense in $H$ by the Lax-Milgram theorem and it is easy to see that

$$
\delta\|u\| \leq\left|A_{H}(t) u\right| \leq\|u\|_{D\left(A_{H}(t)\right)} .
$$

It is obvious that $A(t)$ is an extension of the operator $A_{H}(t)$. Here we note that $D(A(t))=V$ is independent of $t$.

Consider the initial-value problem for the inhomogeneous second order, hyperbolic equation

$$
\left\{\begin{array}{l}
u^{\prime \prime}(t)+A(t) u(t)=f(t), \\
u(0)=u_{0}, \quad u^{\prime}(0)=u_{1}
\end{array}\right.
$$

Put

$$
\mathcal{A}(t)\left(\begin{array}{l}
u_{0} \\
u_{1}
\end{array}\right)=\left(\begin{array}{cc}
0 & -I \\
A(t) & 0
\end{array}\right)\left(\begin{array}{l}
u_{0} \\
u_{1}
\end{array}\right)=\left(\begin{array}{c}
-u_{1} \\
A(t) u_{0}
\end{array}\right) .
$$

Let $U(t)=\left(\begin{array}{l}u_{0}(t) \\ u_{1}(t)\end{array}\right)$ where $u_{1}(t)=\frac{d}{d t} u_{0}(t)$, and let $F(t)=\left(\begin{array}{c}0 \\ f(t)\end{array}\right)$. Then the equation (LE) can be rewritten as

$$
\left\{\begin{array}{l}
U^{\prime}(t)+\mathcal{A}(t) U(t)=F(t) \\
U(0)=U_{0}
\end{array}\right.
$$

where $U_{0}=\left(\begin{array}{l}u_{0} \\ u_{1}\end{array}\right)$. We know that $A_{H}(t)$ and $A(t)$ generate analytic semigroups in $H$ and $V^{*}$, respectively, so the equation (LE) is considered both in $H$ and in $V^{*}$.

Let $X$ be a Banach space. We denoted by $G(X, M, \beta)$ the set of all linear operators $A$ in $X$ such that $A$ generates a $C_{0}$-semigroup $\left\{e^{t A}\right\}$ with

$$
\left\|e^{t A}\right\|_{\mathcal{L}(X)} \leq M e^{\beta t}, \quad 0 \leq t \leq \infty .
$$

We write

$$
G(X) \equiv \bigcup_{M>0, \beta \in \mathbb{R}} G(X, M, \beta)
$$

Definition 2.1. Let $\{A(t): 0 \leq t \leq T\}$ be a family of operators in $G(X) .\{A(t)\}$ is said to be "stable" with "stability index" $M$ and $\beta$ if there are $M>0$ and $\beta \in \mathbb{R}$ such that

$$
\left\|\prod_{j=1}^{k}\left(A\left(t_{j}\right)+\lambda\right)^{-1}\right\|_{\mathcal{L}(X)} \leq M(\lambda-\beta)^{-k}, \quad \lambda>\beta
$$

for every finite family $0 \leq t_{1} \leq t_{2} \leq \cdots \leq t_{k} \leq T, k \in \mathbb{N}$.

The operator product on the left-hand side is time-ordered: $A\left(t_{j}\right)$ is on the left of $A\left(t_{i}\right)$ if $t_{j}>t_{i}$.

Proposition 2.1. For each $t \in[0, T]$, let $\|\cdot\|_{t}$ be a new norm in $X$ equivalent to the original one, depending on $t$ smoothly, in the sense that

$$
\frac{\|x\|_{t}}{\|x\|_{s}} \leq \exp (c|t-s|), \quad x \in X, \quad s, t \in[0, T] .
$$


Assume that for each $t, A(t) \in G\left(X_{t}, 1, \beta\right)$, where $X_{t}$ means the space $X$ with norm $\|\cdot\|_{t}$. Then $\{A(t)\}$ is stable, with respect to $\|\cdot\|_{t}$, for any $t \in[0, T]$, with stability indexes $M \equiv \exp (2 c T)$ and $\beta$, (cf. Proposition 4.3.2 of [15]).

Proposition 2.2 (Corollary in Section 4.4 of [15]). Suppose that $A(t)$ is stable, its domain $D(A(t))=V$ is independent of $t$ for $t \geq 0$ and $A(t) v$ for each $v \in V$ is strongly continuously differentiable on $[0, T]$. Then there exists a unique function $U(t, s) \in \mathcal{L}(X)$ such that $U(t, s)$ maps $V$ into $V, U(t, s) v$, for each $v \in V$, is strongly continuously differentiable in $t$ and $s$, and the following results hold:

(a) $U(t, s)$ is strongly continuous in $s$ and $t, U(s, s)=I$ and $\|U(t, s)\|_{\mathcal{L}(X)} \leq$ $M e^{\beta(t-s)}$

(b) $U(t, s)=U(t, r) U(r, s)$ for $s \leq r \leq t$,

(c) $\partial / \partial t U(t, s) v=-A(t) U(t, s) v$,

(d) $\partial / \partial s U(t, s) v=U(t, s) A(s) v$.

Put $X=(V \times H)^{T}, \tilde{X}=\left(H \times V^{*}\right)^{T}$. We define inner products in $X$ and $\tilde{X}$ by

and

$$
\left(\left(\begin{array}{l}
u_{0} \\
u_{1}
\end{array}\right),\left(\begin{array}{l}
v_{0} \\
v_{1}
\end{array}\right)\right)_{X}=\left(u_{0}, v_{0}\right)+\left(u_{1}, v_{1}\right),
$$

respectively.

$$
\left(\left(\begin{array}{l}
f_{0} \\
f_{1}
\end{array}\right),\left(\begin{array}{l}
g_{0} \\
g_{1}
\end{array}\right)\right)_{\tilde{X}}=\left(f_{0}, g_{0}\right)+\left(f_{1}, g_{1}\right)_{*},
$$

We introduce a new inner product $\langle\cdot, \cdot\rangle_{t}$ and norm $\|\cdot\|_{t}$ into $X$ as

$$
\left\langle\left(\begin{array}{l}
u_{0} \\
u_{1}
\end{array}\right),\left(\begin{array}{l}
v_{0} \\
v_{1}
\end{array}\right)\right\rangle_{t}=a\left(t ; u_{0}, v_{0}\right)+\left(u_{1}, v_{1}\right)
$$

and

$$
\left\|\left(\begin{array}{l}
u_{0} \\
u_{1}
\end{array}\right)\right\|_{t}=\left\{a\left(t ; u_{0}, u_{0}\right)+\left(u_{1}, u_{1}\right)\right\}^{1 / 2}
$$

for $\left(\begin{array}{l}u_{0} \\ u_{1}\end{array}\right),\left(\begin{array}{l}v_{0} \\ v_{1}\end{array}\right) \in X$. Let us introduce a new norm in $V^{*}$ as follows. For $f_{1}, g_{1} \in V^{*}$ we set

$$
\left(f_{1}, g_{1}\right)_{*, t}=a\left(t ; A(t)^{-1} f_{1}, A(t)^{-1} g_{1}\right)=\left(f_{1}, A(t)^{-1} g_{1}\right),
$$

with corresponding norm given by

$$
\left\|f_{1}\right\|_{*, t}=\left(f_{1}, f_{1}\right)_{*, t}^{1 / 2}=a\left(t ; A(t)^{-1} f_{1}, A(t)^{-1} f_{1}\right)^{1 / 2}=\left(f_{1}, A(t)^{-1} f_{1}\right)^{1 / 2} .
$$

It is easily seen that the norm $\|\cdot\|_{*, t}$ is equivalent to $\|\cdot\|_{*}$, i.e, we have

$$
\frac{\delta}{\sqrt{c}_{0}}\|\cdot\|_{*, t} \leq\|\cdot\|_{*} \leq \frac{c_{0}}{\sqrt{\delta}}\|\cdot\|_{*, t} .
$$

We also introduce a inner product $(\cdot, \cdot)_{t}$ and norm $|\cdot|_{t}$ into $\tilde{X}$ as

$$
\begin{aligned}
\left(\left(\begin{array}{l}
f_{0} \\
f_{1}
\end{array}\right),\left(\begin{array}{l}
g_{0} \\
g_{1}
\end{array}\right)\right)_{t} & =\left(f_{0}, g_{0}\right)+a\left(t ; A(t)^{-1} f_{1}, A(t)^{-1} g_{1}\right) \\
& =\left(f_{0}, g_{0}\right)+\left(f_{1}, A(t)^{-1} g_{1}\right)_{*, t}
\end{aligned}
$$


and

$$
\left|\left(\begin{array}{l}
f_{0} \\
f_{1}
\end{array}\right)\right|_{t}=\left(\left|f_{0}\right|^{2}+\left\|f_{1}\right\|_{*, t}^{2}\right)^{1 / 2} .
$$

The Hilbert spaces defined by the inner products mentioned above will be denoted by $X_{t}$ and $\widetilde{X}_{t}$, respectively.

Let $\mathcal{A}_{X}(t)$ be the operator defined by

$$
\begin{aligned}
D\left(\mathcal{A}_{X}(t)\right) & =\left(D\left(A_{H}(t)\right) \times V\right)^{T}, \\
\mathcal{A}_{X}(t)\left(\begin{array}{l}
u_{0} \\
u_{1}
\end{array}\right) & =\left(\begin{array}{cc}
0 & -I \\
A_{H}(t) & 0
\end{array}\right)\left(\begin{array}{l}
u_{0} \\
u_{1}
\end{array}\right)=\left(\begin{array}{c}
-u_{1} \\
A_{H}(t) u_{0}
\end{array}\right) \in(V \times H)^{T}=X .
\end{aligned}
$$

In virtue of Lax-Milgram theorem we can also define $\mathcal{A}(t)$ as

$$
\begin{aligned}
D(\mathcal{A}(t)) & =(V \times H)^{T}=X \\
\mathcal{A}(t)\left(\begin{array}{l}
g_{0} \\
g_{1}
\end{array}\right) & =\left(\begin{array}{cc}
0 & -I \\
A(t) & 0
\end{array}\right)\left(\begin{array}{l}
g_{0} \\
g_{1}
\end{array}\right)=\left(\begin{array}{c}
-g_{1} \\
A(t) g_{0}
\end{array}\right) \in\left(H \times V^{*}\right)^{T}=\tilde{X} .
\end{aligned}
$$

Theorem 2.1. The linear operators $\mathcal{A}_{X}(t)$ and $\mathcal{A}(t)$ mentioned above are the infinitesimal generators of $C_{0}$-groups of unitary operators in $X_{t}$ and $\tilde{X}_{t}$, respectively.

Proof. First we shall prove that $\mathcal{A}_{X}(t)$ and $\mathcal{A}(t)$ are skew self-adjoint operators on $X_{t}$ and $\widetilde{X}_{t}$, respectively. For every $\left(\begin{array}{l}u_{0} \\ u_{1}\end{array}\right),\left(\begin{array}{c}v_{0} \\ v_{1}\end{array}\right) \in D\left(\mathcal{A}_{X}(t)\right)=\left(D\left(A_{H}(t)\right) \times V\right)^{T}$, we have

$$
\begin{aligned}
\left\langle\mathcal{A}_{X}(t)\left(\begin{array}{c}
u_{0} \\
u_{1}
\end{array}\right),\left(\begin{array}{c}
v_{0} \\
v_{1}
\end{array}\right)\right\rangle_{t} & =\left\langle\left(\begin{array}{c}
-u_{1} \\
A_{H}(t) u_{0}
\end{array}\right),\left(\begin{array}{c}
v_{0} \\
v_{1}
\end{array}\right)\right\rangle_{t} \\
& =a\left(t ;-u_{1}, v_{0}\right)+\left(A_{H}(t) u_{0}, v_{1}\right) \\
& =-\left(A_{H}(t) u_{1}, v_{0}\right)+\left(A_{H}(t) u_{0}, v_{1}\right),
\end{aligned}
$$

and

$$
\begin{aligned}
\left\langle\left(\begin{array}{l}
u_{0} \\
u_{1}
\end{array}\right), \mathcal{A}_{X}(t)\left(\begin{array}{c}
v_{0} \\
v_{1}
\end{array}\right)\right\rangle_{t} & =\left\langle\left(\begin{array}{c}
u_{0} \\
u_{1}
\end{array}\right),\left(\begin{array}{c}
-v_{1} \\
A_{H}(t) v_{0}
\end{array}\right)\right\rangle_{t} \\
& =-a\left(t ; u_{0}, v_{1}\right)+\left(u_{1}, A_{H}(t) v_{0}\right) \\
& =-\left(A_{H}(t) u_{0}, v_{1}\right)+\left(u_{1}, A_{H}(t) v_{0}\right)
\end{aligned}
$$

Noting that $A_{H}(t)$ is symmetric, we have that $\mathcal{A}_{X}^{*}(t)=-\mathcal{A}_{X}(t)$, i.e., $i \mathcal{A}_{X}(t)=$ $\left(i \mathcal{A}_{X}(t)\right)^{*}$, therefore, $i \mathcal{A}_{X}(t)$ is self adjoint (and $\mathcal{A}_{X}(t)$ is skew self adjoint). Hence, from Stone's theorem, it follows that $\mathcal{A}_{X}(t)$ is the infinitesimal generator of a $C_{0^{-}}$ group of unitary operators. 
For every $\left(\begin{array}{l}u_{0} \\ u_{1}\end{array}\right),\left(\begin{array}{l}v_{0} \\ v_{1}\end{array}\right) \in \widetilde{X}=V \times H$, we have also that

$$
\begin{aligned}
\left(\mathcal{A}(t)\left(\begin{array}{l}
u_{0} \\
u_{1}
\end{array}\right),\left(\begin{array}{c}
v_{0} \\
v_{1}
\end{array}\right)\right)_{t} & =\left(\left(\begin{array}{c}
-u_{1} \\
A(t) u_{0}
\end{array}\right),\left(\begin{array}{c}
v_{0} \\
v_{1}
\end{array}\right)\right)_{t} \\
& =\left(-u_{1}, v_{0}\right)+\left(A(t) u_{0}, v_{1}\right)_{*} \\
& =-\left(u_{1}, v_{0}\right)+a\left(t ; A(t)^{-1} A(t) u_{0}, A(t)^{-1} v_{1}\right) \\
& =-\left(u_{1}, v_{0}\right)+a\left(t ; u_{0}, A(t)^{-1} v_{1}\right) \\
& =-\left(u_{1}, v_{0}\right)+\overline{a\left(t ; A(t)^{-1} v_{1}, u_{0}\right)} \\
& =-\left(u_{1}, v_{0}\right)+\overline{\left(v_{1}, u_{0}\right)}=-\left(u_{1}, v_{0}\right)+\left(u_{0}, v_{1}\right)
\end{aligned}
$$

and

$$
\begin{aligned}
\left(\left(\begin{array}{l}
u_{0} \\
u_{1}
\end{array}\right), \mathcal{A}(t)\left(\begin{array}{l}
v_{0} \\
v_{1}
\end{array}\right)\right)_{t} & =\left(\left(\begin{array}{c}
u_{0} \\
u_{1}
\end{array}\right),\left(\begin{array}{c}
-v_{1} \\
A(t) v_{0}
\end{array}\right)\right)_{t} \\
& =\left(u_{0},-v_{1}\right)+\left(u_{1}, A(t) v_{0}\right)_{*} \\
& =-\left(u_{0}, v_{1}\right)+a\left(t ; A(t)^{-1} u_{1}, A(t)^{-1} A(t) v_{0}\right) \\
& =-\left(u_{0}, v_{1}\right)+a\left(t ; A(t)^{-1} u_{1}, v_{0}\right)=-\left(u_{0}, v_{1}\right)+\left(u_{1}, v_{0}\right) .
\end{aligned}
$$

Hence,

$$
\left(\mathcal{A}(t)\left(\begin{array}{l}
u_{0} \\
u_{1}
\end{array}\right),\left(\begin{array}{l}
v_{0} \\
v_{1}
\end{array}\right)\right)_{t}=-\left(\left(\begin{array}{l}
u_{0} \\
u_{1}
\end{array}\right), \mathcal{A}(t)\left(\begin{array}{l}
v_{0} \\
v_{1}
\end{array}\right)\right)_{t},
$$

so $\mathcal{A}(t)$ is skew self-adjoint operator on $\widetilde{X}_{t}$.

Theorem 2.2. Assume the hypotheses as in Theorem 2.1. Then $\mathcal{A}_{X}(t)$ and $\mathcal{A}(t)$ are stable on $X$ and $\tilde{X}$, respectively.

Proof. In virtue of Theorem 2.1, we may consider that

$$
\mathcal{A}_{X}(t) \in G\left(X_{t}, 1, \beta\right) \quad\left(\text { or } \quad \mathcal{A}(t) \in G\left(\tilde{X}_{t}, 1, \beta\right)\right) .
$$

For every $\left(\begin{array}{l}u_{0} \\ u_{1}\end{array}\right) \in X$, we have

$$
\begin{aligned}
\left|\frac{\left\|\left(\begin{array}{l}
u_{0} \\
u_{1}
\end{array}\right)\right\|_{t}^{2}}{\left\|\left(\begin{array}{c}
u_{0} \\
u_{1}
\end{array}\right)\right\|_{s}^{2}}-1\right| & =\left|\frac{a\left(t ; u_{0}, u_{0}\right)+\left|u_{1}\right|^{2}}{a\left(s ; u_{0}, u_{0}\right)+\left|u_{1}\right|^{2}}-1\right| \\
& =\left|\frac{a\left(t ; u_{0}, u_{0}\right)-a\left(s ; u_{0}, u_{0}\right)}{a\left(s ; u_{0}, u_{0}\right)+\left|u_{1}\right|^{2}}\right| \\
& \leq \frac{c_{1}(t-s)\left\|u_{0}\right\|^{2}}{\delta\left\|u_{0}\right\|^{2}}=\frac{c_{1}}{\delta}|t-s|,
\end{aligned}
$$

so that

$$
\frac{\left\|\left(\begin{array}{c}
u_{0} \\
u_{1}
\end{array}\right)\right\|_{t}^{2}}{\left\|\left(\begin{array}{c}
u_{0} \\
u_{1}
\end{array}\right)\right\|_{s}^{2}} \leq 1+\frac{c_{1}}{\delta}|t-s| \leq e^{c_{1}|t-s| / \delta} .
$$


Therefore $\left\{\mathcal{A}_{X}(t)\right\}$ is stable with the stability indexes $M=e^{2 c_{1} T / \delta}$ and $\beta=0$ on $X$. For $f \in V^{*}$, we have

$$
\begin{aligned}
\|f\|_{*, t}^{2}-\|f\|_{*, s}^{2} & =\left(f, A(t)^{-1} f\right)-\left(f, A(s)^{-1} f\right) \\
& =\left(f, A(t)^{-1} f-A(s)^{-1} f\right) .
\end{aligned}
$$

Put $v=A(t)^{-1} f-A(s)^{-1} f$. From

$$
\begin{aligned}
\delta\|v\|^{2} & \leq a(t ; v, v)=a\left(t ; A(t)^{-1} f-A(s)^{-1} f, v\right) \\
& =a\left(t ; A(t)^{-1} f, v\right)-a\left(t ; A(s)^{-1} f, v\right)+a\left(s ; A(s)^{-1} f, v\right)-a\left(s ; A(s)^{-1} f, v\right) \\
& =(f, v)-a\left(t ; A(s)^{-1} f, v\right)+a\left(s ; A(s)^{-1} f, v\right)-(f, v) \\
& =-a\left(t ; A(s)^{-1} f, v\right)+a\left(s ; A(s)^{-1} f, v\right) \\
& \leq c_{1}|t-s| \cdot\left\|A(s)^{-1} f\right\| \cdot\|v\|,
\end{aligned}
$$

we have

$$
\begin{aligned}
\delta\|v\| \leq c_{1}|t-s| \cdot\left\|A(s)^{-1} f\right\| & \leq \frac{c_{1}}{\sqrt{\delta}}|t-s| \cdot a\left(s ; A(s)^{-1} f, A(s)^{-1} f\right)^{1 / 2} \\
& =\frac{c_{1}}{\sqrt{\delta}}|t-s| \cdot\|f\|_{*, s} .
\end{aligned}
$$

Therefore, from (2.3), it holds that

$$
\begin{aligned}
\left|\|f\|_{*, t}^{2}-\|f\|_{*, s}^{2}\right| & =|(f, v)| \leq\|f\|_{*}\|v\| \\
& \leq \frac{c_{0}}{\sqrt{\delta}}\|f\|_{*, s} \frac{c_{1}}{\delta \sqrt{\delta}}|t-s| \cdot\|f\|_{*, s} \\
& =\frac{c_{0} c_{1}}{\delta^{2}}|t-s| \cdot\|f\|_{*, s}^{2} .
\end{aligned}
$$

Finally, we have

$$
\begin{aligned}
|| \frac{\left.\left(\begin{array}{c}
f_{0} \\
f_{1}
\end{array}\right)\right|_{t} ^{2}}{\left|\left(\begin{array}{c}
f_{0} \\
f_{1}
\end{array}\right)\right|_{s}^{2}}-1 \mid & =\left|\frac{\left|f_{0}\right|^{2}+\left\|f_{1}\right\|_{*, t}^{2}}{\left|f_{0}\right|^{2}+\left\|f_{1}\right\|_{*, s}^{2}}-1\right| \\
& =\left|\frac{\left\|f_{1}\right\|_{*, t}^{2}-\left\|f_{1}\right\|_{*, s}^{2}}{\left|f_{0}\right|^{2}+\left\|f_{1}\right\|_{*, s}^{2}}\right| \\
& \leq \frac{{ }_{0} c_{1}|t-s| / \delta^{2} \cdot\left\|f_{1}\right\|_{*, s}^{2}}{\left\|f_{1}\right\|_{*, s}^{2}}=c_{0} c_{1}|t-s| / \delta^{2}
\end{aligned}
$$

so $\{\mathcal{A}(t)\}$ is stable with index $M=\exp \left(2 c_{0} c_{1} T / \delta^{2}\right)$ and $\beta=0$ on $\widetilde{X}$.

In virtue of Theorems 2.1, 2.2, we obtain the following results from Propositions $2.1,2.2$.

Theorem 2.3. Let $\mathcal{A}_{X}(t)$ and $\mathcal{A}(t)$ be the operators mentioned above. Then there exist fundamental solutions $\mathcal{U}_{X}(t, s)$ and $\mathcal{U}(t, s)$ satisfying $(\mathrm{a}),(\mathrm{b}),(\mathrm{c})$, and (d) in Proposition 2.2 in $X$ and $\widetilde{X}$, respectively. 
Proof. For every $\left(\begin{array}{l}u_{0} \\ u_{1}\end{array}\right) \in D\left(\mathcal{A}_{X}(t)\right)=D\left(A_{H}(t)\right) \times V$, we have

$$
\frac{d}{d t} \mathcal{A}_{X}(t)\left(\begin{array}{l}
u_{0} \\
u_{1}
\end{array}\right)=\frac{d}{d t}\left(\begin{array}{c}
-u_{1} \\
A_{H}(t) u_{0}
\end{array}\right)=\left(\begin{array}{c}
0 \\
d / d t A_{H}(t) u_{0}
\end{array}\right) .
$$

From this and $\frac{d}{d t}\left(A_{H}(t) u, v\right)=\dot{a}(t ; u, v)$, it follows that $\frac{d}{d t} \mathcal{A}_{X}(t)\left(\begin{array}{l}u_{0} \\ u_{1}\end{array}\right)$ is strongly continuous with respect to $t$, that is, for each $\left(\begin{array}{l}u_{0} \\ u_{1}\end{array}\right) \in D\left(\mathcal{A}_{X}(t)\right)=D\left(A_{H}(t)\right) \times V$ (or $\left.\left(\begin{array}{l}u_{0} \\ u_{1}\end{array}\right) \in X\right), \mathcal{A}_{X}(t)\left(\begin{array}{l}u_{0} \\ u_{1}\end{array}\right)$ (or $\mathcal{A}(t)\left(\begin{array}{l}u_{0} \\ u_{1}\end{array}\right)$, respectively) is strongly continuously differentiable on $[0, T]$. Thus this theorem follows from Theorems 2.1, 2.2 and Proposition 2.2.

\section{Semilinear equations}

Let us first consider existence and regularity of solutions for the following linear inhomogeneous wave equation:

$$
\left\{\begin{array}{l}
u^{\prime \prime}(t)+A(t) u(t)=f(t) \\
u(0)=u_{0}, \quad u^{\prime}(0)=u_{1},
\end{array}\right.
$$

where $A(t)$ satisfies the conditions of the preceding section.

Let $\mathbf{x}(t)=\left(\begin{array}{c}u(t) \\ u^{\prime}(t)\end{array}\right)$ and $F(t)=\left(\begin{array}{c}0 \\ f(t)\end{array}\right)$. We can show that a solution $\mathbf{x}(t)$ of (LE) is represented by

$$
\mathbf{x}(t)=\mathcal{U}(t, 0) \mathbf{x}(0)+\int_{0}^{t} \mathcal{U}(t, s) F(s) d s
$$

where $\mathcal{U}(t, s)$ is the fundamental solution constructed in Theorem 2.3 and Proposition 2.2. Indeed, by Proposition 2.2, we have

$$
\begin{aligned}
\frac{\partial}{\partial s} \mathcal{U}(t, s) \mathbf{x}(s) & =\mathcal{U}(t, s) \mathbf{x}^{\prime}(s)+\mathcal{U}(t, s) \mathcal{A}(s) \mathbf{x}(s) \\
& =\mathcal{U}(t, s)\left(\mathbf{x}^{\prime}(s)+\mathcal{A}(s) \mathbf{x}(s)\right) \\
& =\mathcal{U}(t, s) F(s),
\end{aligned}
$$

which, integrated from 0 to $t$, yields (3.1). Let $T>0$. Define

$$
\begin{aligned}
W_{T} & =\left\{u \mid u \in L^{2}\left(0, T, D\left(A_{H}\right)\right), \dot{u} \in L^{2}(0, T, V), \ddot{u} \in L^{2}(0, T, H)\right\} \\
\|u\|_{W_{T}} & =\|u\|_{L^{2}\left(0, T, D\left(A_{H}\right)\right)}+\|\dot{u}\|_{L^{2}(0, T, V)}+\|\ddot{u}\|_{L^{2}(0, T, H)},
\end{aligned}
$$

and

$$
\begin{aligned}
\widetilde{W}_{T} & =\left\{u \mid u \in L^{2}(0, T, V), \dot{u} \in L^{2}(0, T, H), \ddot{u} \in L^{2}\left(0, T, V^{*}\right)\right\}, \\
\|u\|_{\widetilde{W}_{T}} & =\|u\|_{L^{2}(0, T, V)}+\|\dot{u}\|_{L^{2}(0, T, H)}+\|\ddot{u}\|_{L^{2}\left(0, T, V^{*}\right)},
\end{aligned}
$$

where $\dot{u}$ denote the derivative of $u$ in the generalized sense. Since

$$
\mathcal{A}(t)^{-1}=\left(\begin{array}{cc}
0 & A(t)^{-1} \\
-I & 0
\end{array}\right): \widetilde{X} \rightarrow X
$$


is a bounded operator, we have that $\mathcal{A}(t) \mathcal{U}(t, s) \mathcal{A}(t)^{-1}: \widetilde{X} \rightarrow \widetilde{X}$ is bounded and strong continuous jointly in $s, t$. Therefore, there is constant $M>0$ such that

$$
\|\mathcal{U}(t, s)\|_{\mathcal{L}(\widetilde{X})} \leq M, \quad\left\|\mathcal{A}(t) \mathcal{U}(t, s) \mathcal{A}(s)^{-1}\right\|_{\mathcal{L}(\widetilde{X})} \leq M .
$$

Since $a(s ; u, v)$ satisfies assumption i), it holds that for every $u, v \in V$,

$$
\left|\frac{d}{d s}(A(s) u, v)\right|=|\dot{a}(s ; u, v)| \leq c_{1}\|u\|\|v\|,
$$

that is, we have that for every $u \in V, s \mapsto d / d s A(s) u$ is strongly continuous in $V^{*}$ and so, $\|d / d s A(s)\|_{\mathcal{L}\left(V, V^{*}\right)}$ is bounded on $[0, T]$. Hence, noting that for every $\left(\begin{array}{l}u_{0} \\ u_{1}\end{array}\right) \in X$,

$$
\frac{d}{d s} \mathcal{A}(s)\left(\begin{array}{l}
u_{0} \\
u_{1}
\end{array}\right)=\frac{d}{d s}\left(\begin{array}{c}
-u_{1} \\
A(s) u_{0}
\end{array}\right)=\left(\begin{array}{c}
0 \\
d / d s A(s) u_{0}
\end{array}\right),
$$

it follows that $d / d s \mathcal{A}(s)\left(\begin{array}{l}u_{0} \\ u_{1}\end{array}\right)$ is strongly continuous with respect to $t$ in $\widetilde{X}$ and so, $\|d / d s \mathcal{A}(s)\|_{\mathcal{L}(X, \tilde{X})}$ is bounded on $[0, T]$. Therefore, we may assume that

$$
\left\|\frac{d}{d s} \mathcal{A}(s) \mathcal{A}(s)^{-1}\right\|_{\mathcal{L}(\tilde{X})} \leq M .
$$

Now we prove some energy inequalities for our problem (LE). These will play an important role in the proof of the existence of solution and in the study of the regularity of the solutions.

Theorem 3.1. Assume that $f \in C\left([0, T] ; V^{*}\right) \cap W^{1,2}\left(0, T ; V^{*}\right)(T>0)$ and the initial data $\left(u_{0}, u_{1}\right) \in V \times H$. Then the solution $u$ of (LE) exists and is unique in

$$
u \in \widetilde{W}_{T} \cap C([0, T] ; V) \cap C^{1}([0, T) ; H) .
$$

Furthermore, the following energy inequality holds: there exists a constant $C_{T}$, depending on $T$, such that

$$
\|u\|_{\widetilde{W}_{T}} \leq C_{T}\left(\left\|u_{0}\right\|+\left|u_{1}\right|+\|f(0)\|_{*}+\|f\|_{W^{1,2}\left(0, T ; V^{*}\right)}\right) .
$$

If $f \in C([0, T] ; H) \cap W^{1,2}(0, T ; H)$ and $\left(u_{0}, u_{1}\right) \in D\left(A_{H}\right) \times V$, then the solution $u$ of (LE) exists, is unique in

$$
u \in W_{T} \cap C\left([0, T] ; D\left(A_{H}\right)\right) \cap C^{1}([0, T) ; V),
$$

and satisfies

$$
\|u\|_{W_{T}} \leq C_{T}\left(\left\|u_{0}\right\|_{D\left(A_{H}\right)}+\left\|u_{1}\right\|+|f(0)|+\|f\|_{W^{1,2}(0, T ; H)}\right) .
$$

Proof. Equation (LE) may be considered as an equation both in $H$ and in $V^{*}$. We now investigate the consequences of the equation considered in $\widetilde{X}$. Since $\left\{\mathcal{A}_{X}(t) \mid\right.$ $0 \leq t \leq T\}$ and $\{\mathcal{A}(t) \mid 0 \leq t \leq T\}$ are stable on $X$ and $\widetilde{X}$, respectively in virtue of Theorem 2.3, there exists a fundamental solution $\mathcal{U}(t, s)$ of

$$
\frac{d}{d t}\left(\begin{array}{l}
w_{0}(t) \\
w_{1}(t)
\end{array}\right)+\mathcal{A}(t)\left(\begin{array}{l}
w_{0}(t) \\
w_{1}(t)
\end{array}\right)=\left(\begin{array}{c}
0 \\
f(t)
\end{array}\right) .
$$


Let $u(t)$ be the solution of the equation

$$
\left\{\begin{array}{l}
u^{\prime \prime}(t)+A(t) u(t)=f(t) \\
u(0)=u_{0}, \quad u^{\prime}(0)=u_{1} .
\end{array}\right.
$$

Then we put $w_{0}(t)=u(t), w_{1}(t)=(d / d t) u(t)$ and hence obtain

$$
\left(\begin{array}{l}
w_{0}(t) \\
w_{1}(t)
\end{array}\right)=\mathcal{U}(t, 0)\left(\begin{array}{l}
w_{0}(0) \\
w_{1}(0)
\end{array}\right)+\int_{0}^{t} \mathcal{U}(t, s)\left(\begin{array}{c}
0 \\
f(s)
\end{array}\right) d s .
$$

From the property (d) in Proposition 2.2, it follows

$$
\begin{aligned}
\int_{0}^{t} \mathcal{U}(t, s)\left(\begin{array}{c}
0 \\
f(s)
\end{array}\right) d s= & \int_{0}^{t} \mathcal{U}(t, s) \mathcal{A}(s) \mathcal{A}(s)^{-1}\left(\begin{array}{c}
0 \\
f(s)
\end{array}\right) d s \\
= & \int_{0}^{t} \frac{\partial}{\partial s} \mathcal{U}(t, s) \mathcal{A}(s)^{-1}\left(\begin{array}{c}
0 \\
f(s)
\end{array}\right) d s \\
= & \mathcal{A}(t)^{-1}\left(\begin{array}{c}
0 \\
f(t)
\end{array}\right)-\mathcal{U}(t, 0) \mathcal{A}(0)^{-1}\left(\begin{array}{c}
0 \\
f(0)
\end{array}\right) \\
& -\int_{0}^{t} \mathcal{U}(t, s) \mathcal{A}(s)^{-1} \\
& \times\left\{-\frac{d}{d s} \mathcal{A}(s) \mathcal{A}(s)^{-1}\left(\begin{array}{c}
0 \\
f(s)
\end{array}\right)+\left(\begin{array}{c}
0 \\
f^{\prime}(s)
\end{array}\right)\right\} d s .
\end{aligned}
$$

From this and (3.6) we have

$$
\begin{aligned}
\mathcal{A}(t)\left(\begin{array}{l}
w_{0}(t) \\
w_{1}(t)
\end{array}\right)= & \mathcal{A}(t) \mathcal{U}(t, 0) \mathcal{A}(0)^{-1} \mathcal{A}(0)\left(\begin{array}{l}
w_{0}(0) \\
w_{1}(0)
\end{array}\right) \\
& +\left(\begin{array}{c}
0 \\
f(t)
\end{array}\right)-\mathcal{A}(t) \mathcal{U}(t, 0) \mathcal{A}(0)^{-1}\left(\begin{array}{c}
0 \\
f(0)
\end{array}\right) \\
& -\int_{0}^{t} \mathcal{A}(t) \mathcal{U}(t, s) \mathcal{A}(s)^{-1} \\
& \times\left\{-\frac{d}{d s} \mathcal{A}(s) \mathcal{A}(s)^{-1}\left(\begin{array}{c}
0 \\
f(s)
\end{array}\right)+\left(\begin{array}{c}
0 \\
f^{\prime}(s)
\end{array}\right)\right\} d s .
\end{aligned}
$$

Therefore, by (3.2) we have that there exists a constant $c_{1}$ such that

$$
\begin{aligned}
\left\|\mathcal{A}(t)\left(\begin{array}{l}
w_{0}(t) \\
w_{1}(t)
\end{array}\right)\right\|_{\tilde{X}} \leq & c_{1}\left\{\left\|\mathcal{A}(0)\left(\begin{array}{l}
w_{0}(0) \\
w_{1}(0)
\end{array}\right)\right\|+\left\|\left(\begin{array}{c}
0 \\
f(t)
\end{array}\right)\right\|\right. \\
& \left.+\left\|\left(\begin{array}{c}
0 \\
f(0)
\end{array}\right)\right\|+\int_{0}^{t}\left\|\left(\begin{array}{c}
0 \\
f(s)
\end{array}\right)\right\|+\left\|\left(\begin{array}{c}
0 \\
f^{\prime}(s)
\end{array}\right)\right\| d s\right\}
\end{aligned}
$$




$$
\begin{aligned}
= & c_{1}\left\{\left\|\left(\begin{array}{c}
-w_{1}(0) \\
A(0) w_{0}(0)
\end{array}\right)\right\|+\|f(t)\|_{*}+\|f(0)\|_{*}\right. \\
& \left.+\int_{0}^{t}\left(\|f(s)\|_{*}+\left\|f^{\prime}(s)\right\|_{*}\right) d s\right\},
\end{aligned}
$$

where $c_{1}=\max \left\{M, 1, M^{2}\right\}$. Here, we remark that

$$
\begin{aligned}
\left\|\mathcal{A}(0)\left(\begin{array}{c}
w_{0}(0) \\
w_{1}(0)
\end{array}\right)\right\|_{\tilde{X}} & =\left\|\left(\begin{array}{c}
-w_{1}(0) \\
A(0) w_{0}(0)
\end{array}\right)\right\|_{\tilde{X}} \\
& =\left(\left|w_{1}(0)\right|^{2}+\left\|A(0) w_{0}(0)\right\|_{*}^{2}\right)^{\frac{1}{2}} \\
& \leq \max \left\{1, c_{0}\right\}\left(\left\|w_{0}(0)\right\|+\left|w_{1}(0)\right|\right),
\end{aligned}
$$

that

$$
\|f(t)\|_{*}=\left\|f(0)+\int_{0}^{t} f^{\prime}(s) d s\right\|_{*} \leq\left\|f_{0}\right\|_{*}+\int_{0}^{t}\left\|f^{\prime}(s)\right\|_{*} d s
$$

and

$$
\begin{aligned}
\int_{0}^{t}\|f(s)\|_{*} d s & =\int_{0}^{t}\left\|f(0)+\int_{0}^{s} f^{\prime}(\sigma) d \sigma\right\|_{*} d s \\
& \leq t\|f(0)\|_{*}+\int_{0}^{t} \int_{0}^{s}\left\|f^{\prime}(\sigma)\right\|_{*} d \sigma d s \\
& =t\|f(0)\|_{*}+\int_{0}^{t}(t-\sigma)\left\|f^{\prime}(\sigma)\right\|_{*} d \sigma .
\end{aligned}
$$

We recall that

$$
\left\|\mathcal{A}(t)\left(\begin{array}{l}
w_{0}(t) \\
w_{1}(t)
\end{array}\right)\right\|_{\tilde{X}} \geq \min \{1, \delta\}\left(\left\|w_{0}(t)\right\|^{2}+\left|w_{1}(t)\right|^{2}\right)^{\frac{1}{2}} .
$$

Hence, from (3.6)-(3.12), it follows that

$$
\begin{aligned}
& \left(\left\|w_{0}(t)\right\|^{2}+\left|w_{1}(t)\right|^{2}\right)^{1 / 2} \leq c_{1} / \min \{1, \delta\} \times \\
& \quad \times\left\{\max \left\{1, c_{0}\right\}\left(\left\|w_{0}(0)\right\|+\left|w_{1}(0)\right|\right)+\|f(0)\|_{*}+\int_{0}^{t}\left\|f^{\prime}(s)\right\|_{*} d s\right\} .
\end{aligned}
$$

Therefore, we see that $u \in C([0, T] ; V) \cap C^{1}([0, T) ; H)$ (the continuity of solutions of (LE) can also be obtained by an application of the theory of intermediate spaces (see [13, Vol. I, Theorem 3.1])). Since $w_{0}(t)=u(t), w_{1}(t)=(d / d t) u(t)$ and $d^{2} / d t^{2} u(t)=-A(t) u(t)+f(t)$, it holds

$$
\left\|\frac{d^{2}}{d t^{2}} u(t)\right\|_{*}=\|-A(t) u(t)+f(t)\|_{*} \leq c_{0}\|u(t)\|+\|f(t)\|_{*} .
$$

By this and (3.13), there exists a constant $C_{T}$ such that

$$
\|u\|_{\widetilde{W}_{T}} \leq C_{T}\left(\left\|u_{0}\right\|+\left|u_{1}\right|+\|f(0)\|_{*}+\|f\|_{W^{1,2}\left(0, T ; V^{*}\right)}\right) .
$$


Let $f \in C([0, T] ; H) \cap W^{1,2}(0, T ; H)(T>0)$ and $\left(u_{0}, u_{1}\right) \in D\left(A_{H}\right) \times V$. Consider the equation (LE) in $X$. Then the estimate (3.5) on $W_{T}$ is completely analogous to that on $\widetilde{W}_{T}$ in $\widetilde{X}$.

From now on, using the properties of the linear inhomogeneous equations, we investigate the regularity of solutions for abstract semilinear second order initial value problem:

$$
\left\{\begin{array}{l}
u^{\prime \prime}(t)+A(t) u(t)=G(t, u(t))+f(t) \\
u(0)=u_{0}, \quad u^{\prime}(0)=u_{1} .
\end{array}\right.
$$

We assume the following hypotheses on the nonlinear term.

Assumption (G). Let $G:[0, T] \times V \rightarrow H$ be a nonlinear mapping such that $t \mapsto$ $G(t, \cdot)$ is continuously differentiable on $[0, T]$ and $u \mapsto G(\cdot, u)$ is Lipschitz continuous on $V$. Then there exists a constant $L>0$ such that

$$
\begin{aligned}
\sup _{0 \leq t \leq T}\left|\frac{\partial}{\partial t} G(t, u)\right| & \leq L, \\
|G(\cdot, u)-G(\cdot, v)| & \leq L\|u-v\|, \quad u, v \in V .
\end{aligned}
$$

In case where $A=A(t)$, by Theorem 3.1 in [7] (or Theorem 6.1.3 in [15]), the problems (SE) has a unique local solution on some interval $\left[0, T_{c}\right)$ for some $T_{c} \leq T$. Also when $A(t)$ depends on $t$, similar results hold when the equation (SE) has a fundamental solution, see Remark 6.1 .1 of [15]. We shall see that the solution can be extended to $[0, T]$ for $T>0$. To see this, it is enough to show that if $u$ is a solution in $0<T_{c} \leq T<\infty$, then $u(t)$ is bounded in $0 \leq t<T_{c}$. We start with the following results.

Theorem 3.2. Let Assumption $(\mathrm{G})$ be satisfied. Assume that $f \in C\left([0, T] ; V^{*}\right) \cap$ $W^{1,2}\left(0, T ; V^{*}\right)(T>0)$ and $\left(u_{0}, u_{1}\right) \in V \times H$. Then the solution $u$ of (SE) exists and is unique in

$$
u \in \widetilde{W}_{T} \cap C([0, T] ; V) \cap C^{1}([0, T) ; H), \quad T>0 .
$$

Furthermore, the following energy inequality holds: there exists a constant $C_{T}$, depending on $T$, such that

$$
\|u\|_{\widetilde{W}_{T}} \leq C_{T}\left(1+\left\|u_{0}\right\|+\left|u_{1}\right|+\|f(0)\|_{*}+\|f\|_{W^{1,2}\left(0, T ; V^{*}\right)}\right) .
$$

Proof. Let $u(t)$ be the solution of the following equation

$$
\left\{\begin{array}{l}
u^{\prime \prime}(t)+A(t) u(t)=G(t, u(t))+f(t) \\
u(0)=u_{0}, \quad u^{\prime}(0)=u_{1} .
\end{array}\right.
$$

Put $w_{0}=u(t), w_{1}(t)=u^{\prime}(t)$. Then, by Theorem 2.3, there exists a fundamental solution $\mathcal{U}(t, s)$ of

$$
\frac{d}{d t}\left(\begin{array}{l}
w_{0}(t) \\
w_{1}(t)
\end{array}\right)+\mathcal{A}(t)\left(\begin{array}{l}
w_{0}(t) \\
w_{1}(t)
\end{array}\right)=\left(\begin{array}{c}
0 \\
G\left(t, w_{0}(t)\right)+f(t)
\end{array}\right)
$$


and hence obtain

$$
\left(\begin{array}{l}
w_{0}(t) \\
w_{1}(t)
\end{array}\right)=\mathcal{U}(t, 0)\left(\begin{array}{l}
w_{0}(0) \\
w_{1}(0)
\end{array}\right)+\int_{0}^{t} \mathcal{U}(t, s)\left(\begin{array}{c}
0 \\
G\left(t, w_{0}(t)\right)+f(t)
\end{array}\right) d s .
$$

From (3.15), as in the argument of (3.7), we have

$$
\begin{aligned}
\mathcal{A}(t)\left(\begin{array}{l}
w_{0}(t) \\
w_{1}(t)
\end{array}\right)= & \mathcal{A}(t) \mathcal{U}(t, 0) \mathcal{A}(0)^{-1} \mathcal{A}(0)\left(\begin{array}{l}
w_{0}(t) \\
w_{1}(t)
\end{array}\right) \\
& +\left(\begin{array}{c}
0 \\
G\left(t, w_{0}(t)\right)+f(t)
\end{array}\right)-\mathcal{A}(t) \mathcal{U}(t, 0) \mathcal{A}(0)^{-1}\left(\begin{array}{c}
0 \\
G\left(0, w_{0}(0)\right)+f(0)
\end{array}\right) \\
& -\int_{0}^{t} \mathcal{A}(t) \mathcal{U}(t, s) \mathcal{A}(s)^{-1}\left\{-\frac{d}{d s} \mathcal{A}(s) \mathcal{A}(s)^{-1}\left(\begin{array}{c}
0 \\
G\left(s, w_{0}(s)\right)+f(s)
\end{array}\right)\right. \\
& \left.+\left(\begin{array}{c}
0 \\
\frac{d}{d s}\left(G\left(s, w_{0}(s)\right)+f(s)\right.
\end{array}\right)\right\} d s .
\end{aligned}
$$

Furthermore, as in (3.8), there exists a constant $c_{1}$ such that

$$
\begin{aligned}
\| \mathcal{A}(t) & \left(\begin{array}{l}
w_{0}(t) \\
w_{1}(t)
\end{array}\right) \| \leq \\
c_{1} & \left\{\left\|\left(\begin{array}{c}
-w_{1}(0) \\
A(0) w_{0}(0)
\end{array}\right)\right\|+\left|G\left(t, w_{0}(t)\right)+f(t)\right|_{*}+\left|G\left(0, w_{0}(0)\right)+f(0)\right|_{*}\right. \\
& \left.+\int_{0}^{t}\left(\left\|G\left(s, w_{0}(s)\right)+f(s)\right\|_{*}+\left\|\frac{d}{d s} G\left(s, w_{0}(s)\right)+f(s)\right\|_{*}\right) d s\right\} .
\end{aligned}
$$

Now, noting that

$$
\begin{aligned}
\left\|\frac{d}{d s} G\left(s, w_{0}(s)\right)\right\|_{*} & =\left\|G_{1}\left(s, w_{0}(s)\right)+G_{2}\left(s, w_{0}(s)\right) u^{\prime}(s)\right\|^{*} \\
& \leq\left\|G_{1}\left(s, w_{0}(s)\right)\right\|_{*}+L\left\|w_{0}^{\prime}(s)\right\|
\end{aligned}
$$

where $G_{i}(i=1,2)$ denote the partial derivatives of $G$, we have

$$
\begin{aligned}
\| G\left(t, w_{0}(t) \|_{*}=\right. & \left\|G\left(0, w_{0}(0)\right)+\int_{0}^{t} \frac{d}{d s} G\left(s, w_{0}(s)\right) d s\right\|_{*} \\
\leq & \left(\left|G\left(0, w_{0}(0)\right)-G(0,0)\right|+|G(0,0)|\right. \\
& +\int_{0}^{t}\left(\left|G_{1}\left(s, w_{0}(s)\right)\right|+L\left\|w_{0}^{\prime}(s)\right\| d s\right) \\
\leq & L\left\|w_{0}(0)\right\|+|G(0,0)|+L \int_{0}^{t}\left(1+\left\|w_{0}^{\prime}(s)\right\|\right) d s
\end{aligned}
$$


and

$$
\begin{aligned}
\int_{0}^{t} \| G\left(s, w_{0}(s) \|_{*} d s\right. & =\int_{0}^{t}\left\|G\left(0, w_{0}(0)\right)+\int_{0}^{s} \frac{d}{d \sigma} G\left(\sigma, w_{0}(\sigma)\right) d \sigma\right\|_{*} d s \\
& \leq t \| G\left(0, w_{0}(0)\left\|_{*}+\int_{0}^{t}(t-\sigma)\right\| \frac{d}{d \sigma} G\left(\sigma, w_{0}(\sigma)\right) \|_{*} d s\right. \\
& \leq t \| G\left(0, w_{0}(0) \|_{*}+L \int_{0}^{t}(t-\sigma)\left(1+\left\|w_{0}^{\prime}(\sigma)\right\|\right) d \sigma .\right.
\end{aligned}
$$

Thus, from (3.9), (3.11), (3.16)-(3.19), we have that there exists a constant $C^{\prime}$ depending on $T$ such that

$$
\begin{aligned}
\left(\left\|w_{0}(t)\right\|^{2}+\left|w_{1}(t)\right|^{2}\right)^{1 / 2} \leq & C^{\prime}\left(1+\left\|w_{0}\right\|+\left|w_{1}\right|+\|f(0)\|_{*}\right. \\
& \left.+\|f\|_{W^{1,2}\left(0, T ; V^{*}\right)}+\int_{0}^{t}\left(1+\left\|w_{0}^{\prime}(s)\right\|\right) d s\right),
\end{aligned}
$$

noting that $w_{1}(t)=(d / d t) u(t)$, which by Gronwall's inequality implies (3.14).

\section{Applications}

Let $\Omega$ be bounded domain in $\mathbb{R}^{n}$ with smooth boundary $\partial \Omega$. We define the following spaces:

$$
\begin{aligned}
& H^{1}(\Omega)=\left\{u \mid u, \frac{\partial u}{\partial x_{i}} \in L^{2}(\Omega), \quad i=1,2, \ldots, n\right\} \\
& H^{2}(\Omega)=\left\{u \mid u, \frac{\partial u}{\partial x_{i}}, \frac{\partial^{2} u}{\partial x_{i} \partial x_{j}} \in L^{2}(\Omega), \quad i, j=1,2, \ldots, n\right\}
\end{aligned}
$$

where $\frac{\partial u}{\partial x_{i}}$ and $\frac{\partial^{2} u}{\partial x_{i} \partial x_{j}}$ are the derivative of $u$ in the distribution sense. The norm of $H^{1}(\Omega)$ is defined by

$$
\|u\|_{1}=\left\{\int_{\Omega}\left(u(x)^{2}+\sum_{i=1}^{n}\left(\frac{\partial u(x)}{\partial x_{i}}\right)^{2}\right) d x\right\}^{1 / 2} .
$$

Hence $H^{1}(\Omega)$ is a Hilbert space.

$$
H_{0}^{1}(\Omega)=\left\{u\left|u \in H^{1}(\Omega), u\right|_{\partial \Omega}=0\right\}=\text { the closure of } C_{0}^{\infty}(\Omega) \text { in } H^{1}(\Omega) .
$$

The norm of $H_{0}^{1}(\Omega)$ is defined by

$$
\|u\|=\left\{\int_{\Omega} \sum_{i=1}^{n}\left(\frac{\partial u(x)}{\partial x_{i}}\right)^{2} d x\right\}^{1 / 2}=\|u\|_{1}
$$

for any $u \in H_{0}^{1}(\Omega)$. Let $H^{-1}(\Omega)=H_{0}^{1}(\Omega)^{*}$ be the dual space of $H_{0}^{1}(\Omega)$. For any $l \in$ $H^{-1}(\Omega)$ and $v \in H_{0}^{1}(\Omega)$, the notation $(l, v)$ denotes the value $l$ at $v$. In what follows, we consider the regularity for given equations, applying the result of Section 3 with $L^{2}(\Omega)$ taking the place of $H$ and $H_{0}^{1}(\Omega)=\left\{u \in H^{1}(\Omega) \mid u=0\right.$ on $\left.\partial \Omega\right\}$ replacing $V$. 
For each $t \in[0, T]$ and $u, v \in H^{1}(\Omega)$, let us consider the following sesquilinear form:

$$
a(t ; u, v)=\sum_{i, j=1}^{n} \int_{\Omega}\left(a_{i j}(t, x) \frac{\partial u}{\partial x_{i}} \frac{\overline{\partial v}}{\partial x_{j}}+c(t, x) u \bar{v}\right) d x
$$

where the matrix $\left(a_{i j}(t, x)\right)$ is uniformly positive definite, i.e., there exists a positive constant $\delta$ such that

$$
\sum_{i, j=1}^{n} a_{i j}(t, x) \xi_{i} \bar{\xi}_{j} \geq \delta|\xi|^{2}
$$

for all $x \in \Omega t \in[0, T]$ and for all real vectors $\xi$. Let

$$
a_{i j}, \quad \frac{\partial}{\partial x_{j}} a_{i j}, \quad \frac{\partial}{\partial t} a_{i j}, \quad \frac{\partial^{2}}{\partial t \partial x_{j}} a_{i j}, \quad c \geq 0, \quad \frac{\partial}{\partial t} c
$$

be all continuous and bounded on $\Omega \times[0, T]$, and

$$
a_{i j}, \quad \frac{\partial}{\partial x_{j}} a_{i j}, \quad c
$$

satisfy uniformly Lipschitz continuity with respect to $t$. Then there exist constants $c_{0}, c_{1}>0$ such that

$$
\begin{aligned}
|a(t, u, v)| & \leq c_{0}\|u\|\|v\| \\
\left|\frac{d}{d t} a(t, u, v)\right| & =\left|\int_{\Omega}\left(\sum_{i, j=1}^{n} \dot{a}_{i j}(t, x) \frac{\partial u}{\partial x_{i}} \overline{\frac{\partial v}{\partial x_{j}}}+\dot{c}(t, x) u \bar{v}\right) d x\right| \leq c_{1}\|u\| \cdot\|v\|
\end{aligned}
$$

and it holds Gårding's inequality:

$$
\begin{aligned}
a(t ; u, u) & =\int_{\Omega}\left(\sum_{i, j=1}^{n} a_{i j}(t, x) \frac{\partial u}{\partial x_{i}} \frac{\overline{\partial u}}{\partial x_{j}}+c(t, x) u \bar{u}\right) d x \\
& \geq \delta \int_{\Omega}\left|\frac{\partial u}{\partial x_{i}}\right|^{2} d x=\delta\|u\|^{2} .
\end{aligned}
$$

Consider the Cauchy problem for the hyperbolic equation:

$$
\left\{\begin{array}{l}
\frac{\partial^{2}}{\partial t^{2}} u(t, x)-\sum_{i, j=1}^{n} \frac{\partial}{\partial x_{j}}\left(a_{i j}(t, x) \frac{\partial u}{\partial x_{i}}\right)+c(t, x) u \\
=\int_{0}^{t} \sum_{i=1}^{n} \frac{\partial}{\partial x_{i}} \sigma_{i}(s, \nabla u(s, x)) d s+h(t), \quad 0 \leq t, \quad x \in \Omega, \\
u(t, x)=0, \quad 0 \leq t, \quad x \in \partial \Omega, \\
u(0, x)=u_{0}(x), \quad \frac{\partial}{\partial t} u(0, x)=u_{1}(x), \quad x \in \Omega .
\end{array}\right.
$$

Define the operator $A(t)$ by

$$
\begin{aligned}
(A(t) u, v) & =a(t ; u, v)=\int_{\Omega}\left(\sum_{i, j=1}^{n} a_{i j}(t, x) \frac{\partial u}{\partial x_{i}} \frac{\overline{\partial v}}{\partial x_{j}}+c(t, x) u \bar{v}\right) d x, \\
D(A(t)) & =\left\{u \mid u \in H^{2}(\Omega) \cap H_{0}^{1}(\Omega)\right\}=\left\{u\left|u \in H^{2}(\Omega), u\right|_{\partial \Omega}=0\right\} .
\end{aligned}
$$


The operator $A(t)$ in $L^{2}(\Omega)$ is defined as follows: for any $v \in H_{0}^{1}(\Omega)$, there exists $f \in L^{2}(\Omega)$ such that

$$
a(t ; u, v)=(f, v) .
$$

We set, for any $u \in D(A(t)), A(t) u=f . A(t)$ is a positive definite self-adjoint operator. For fixed $u$, the functional $H_{0}^{1}(\Omega) \ni v \rightarrow a(t ; u, v)$ is linear and continuous. Then there is $l \in H^{-1}(\Omega)$ such that $(l, v)=a(t ; u, v)$, for all $v$. Setting $\widetilde{A}(t) u=l$ we have that, for any $u, v \in H_{0}^{1}(\Omega)$,

$$
a(t ; u, v)=(\widetilde{A}(t) u, v) .
$$

The operator $\widetilde{A}(t)$ is a one to one mapping from $H_{0}^{1}(\Omega)$ to $H^{-1}(\Omega)$. We have that $A(t)$ and $\widetilde{A} t$ satisfy the following relation:

$$
\begin{aligned}
D(A(t)) & =\left\{u \in H_{0}^{1}(\Omega), \widetilde{A}(t) u \in L^{2}(\Omega)\right\} \\
A(t) u & =\widetilde{A}(t) u \text { for any } u \in D(A(t)) .
\end{aligned}
$$

From now on, both $A(t)$ and $\widetilde{A}(t)$ are denoted simply by $A(t)$. For any $u \in D(A(t))$, we let

$$
G(t, u(t, x))=\int_{0}^{t} \sum_{i=1}^{n} \frac{\partial}{\partial x_{i}} \sigma_{i}(s, \nabla u(s, x)) d s .
$$

Then (4.1) can be seen as the initial value problem for the abstract second order equations:

$$
\left\{\begin{array}{l}
\frac{d^{2}}{d t^{2}} u(t)+A(t) u(t)=G(t, u(t))+f(t) \\
u(0)=u_{0}, \quad \frac{d}{d t} u(0)=u_{1}
\end{array}\right.
$$

We assume the following:

Assumption (G1). The partial derivatives $\sigma_{i}(s, \xi), \partial / \partial t \sigma_{i}(s, \xi)$ and $\partial / \partial \xi_{j} \sigma_{i}(s, \xi)$ exist and are continuous for $i=1,2, j=1,2, \ldots, n$, and $\sigma_{i}(s, \xi)$ satisfies an uniform Lipschitz condition with respect to $\xi$, that is, there exists a constant $L>0$ such that

$$
\left|\sigma_{i}(s, \xi)-\sigma_{i}(s, \widehat{\xi})\right| \leq L|\xi-\widehat{\xi}|
$$

where $|\cdot|$ denotes the norm of $L^{2}(\Omega)$.

Lemma 4.1. If Assumption (G1) is satisfied, then the mapping $t \mapsto G(t, \cdot)$ is continuously differentiable on $[0, T]$ and $u \mapsto G(\cdot, u)$ is Lipschitz continuous on $V$.

Proof. Put

$$
g(s, u)=\sum_{i=1}^{n} \frac{\partial}{\partial x_{i}} \sigma_{i}(s, \nabla u) .
$$

Then we have $g(s, u) \in H^{-1}(\Omega)$. For each $w \in H_{0}^{1}(\Omega)$, we have that

$$
(g(s, u), w)=-\sum_{i=1}^{n}\left(\sigma_{i}(s, \nabla u), \frac{\partial}{\partial x_{i}} w\right) .
$$


The nonlinear term is given by

$$
G(t, u)=\int_{0}^{t} g(s, u) d s .
$$

For any $w \in H_{0}^{1}(\Omega)$, if $u$ and $\hat{u}$ belong to $H_{0}^{1}(\Omega)$, by Assumption (G1) we obtain

$$
|(G(t, u)-G(t, \hat{u})), w| \leq L T\|u-\hat{u}\|\|w\| \text {. }
$$

Now in virtue of Lemma 4.1, we can apply the results of Theorem 3.2 to obtain:

Theorem 4.1. Let Assumption (G1) be satisfied. Assume that

$$
f \in C\left([0, T] ; H^{-1}(\Omega)\right) \cap W^{1,2}\left(0, T ; H^{-1}(\Omega)\right), \quad T>0
$$

and $\left(u_{0}, u_{1}\right) \in H_{0}^{1}(\Omega) \times L^{2}(\Omega)$. Then the solution $u$ of (4.1) exists and is unique in

where

$$
u \in \widetilde{W}_{T} \cap C\left([0, T] ; H_{0}^{1}(\Omega)\right) \cap C^{1}\left([0, T) ; L^{2}(\Omega)\right), \quad T>0
$$

$$
\widetilde{W}_{T}=L^{2}\left(0, T ; H_{0}^{1}(\Omega)\right) \cap W^{1,2}\left(0, T ; L^{2}(\Omega)\right) \cap W^{2,2}\left(0, T ; H^{-1}(\Omega)\right) .
$$

Furthermore, the following energy inequality holds: there exists a constant $C_{T}$ depending on $T$ such that

$$
\|u\|_{\widetilde{W}_{T}} \leq C_{T}\left(1+\left\|u_{0}\right\|+\left|u_{1}\right|+\|f(0)\|_{*}+\|f\|_{W^{1,2}\left(0, T ; H^{-1}(\Omega)\right)}\right) .
$$

\section{Acknowledgements}

The authors wishes to thank the referees for careful reading of manuscript, for valuable suggestions and many useful comments.

\section{References}

[1] V. Barbu, Analysis and Control of Nonlinear Infinite Dimensional Systems, Academic Press Limited, 1993.

[2] W. S. Edelstein and M. E. Gurtin, Uniqueness theorem in the linear dynamic theory of anisotropic viscoelastic solid, Arch. Rat. Mech. Anal., 17 (1964), 47-60.

[3] M. J. Garrido-Atienza and José Real, Existence and uniqueness of solutions for delay evolution equations of second order in time, J. Math. Anal. Appl., 283 (2003), 582609.

[4] M. L. Heard, An abstract parabolic Volterra integro-differential equation, J. Appl. Math., 17 (1981), 175-202.

[5] M. Ikawa, Mixed problems for hyperbolic equations of second order, J. Math. Soc. Japan, 20(4) (1968), 580-608.

[6] J. M. Jeong, Y.C. Kwun and J.Y. Park, Approximate controllability for semilinear retarded functional differential equations, J. Dynamics and Control Systems, 3 (1999), 329-346. 
[7] J.-M. Jeong, J.-R. Kim and H.-G. Kim, Regularity for solutions of nonlinear second order evolution equations, J. Math. Anal. Appl., 17 (2008), 209-222.

[8] A. G. Kartsatos and L. P. Markov, An $L_{2}$-approach to second-order nonlinear functional evolutions involving $m$-accretive operators in Banach spaces, Differential Integral Equations, 14 (2001), 833-866.

[9] T. Kato, Linear evolution equations of "hyperbolic" type, J. Fac. Sci. Univ. Tokyo I, 17(1970), 241-258.

[10] T. Kato, Linear evolution equations of "hyperbolic" type II, J. Math. Soc. Japan, 25 (1973), 638-666.

[11] Y. Kobayashi, T. Matsumoto and N. Tanaka, Semigroups of locally Lipschitz operators associated with semilinear evolution equations, J. Math. Anal. Appl., 330(2) (2007), 1042-1067.

[12] K. Kobayasi, On a theorem for linear evolution equations of hyperbolic type, J. Math. Soc. Japan, 31 (1979), 647-654.

[13] J. L. Lions and E. Magenes, Non-Homogeneous Boundary Value Problems and Applications, Springer Verlag, Berlin, Heidelberg, New York, 1972.

[14] R. H. Martin, Nonlinear operators and differential equations in Banach spaces, John Wiley and Sons, New York, 1976.

[15] H. Tanabe, Equations of Evolution, Pitman London, 1979.

[16] N. Tanaka, Nonautonomous abstract Cauchy problems for strongly measurable families, Math. Nachr., 274/275 (2004), 130-153.

[17] G. Webb, Continuous nonlinear perturbations of linear accretive operators in Banach spaces, J. Fun. Anal., 10 (1972), 191-203.

[18] J. Wu, Theory and Applications of Partial Functional Differential Equations, Springer Verlag, New York, 1996.

Dong-Gun Park

Mathematics and Materials Physics

Dong-A University

Saha-Gu, Busan 604-714

Korea

e-mail: dgpark@dau.ac.kr

Jin-Mun Jeong and Han-Geul Kim

Division of Mathematical Sciences

Pukyong National University

Busan 608-737

Korea

e-mail: jmjeong@pknu.ac.kr

hgkim@hanmail.net

Received: 18 February 2008.

Accepted: 29 October 2008. 